\title{
Real-time confinement following a quantum quench to a non-integrable model
}

\author{
Marton Kormos ${ }^{1}$, Mario Collura ${ }^{2}$, Gabor Takács ${ }^{1,3}$ and Pasquale Calabrese ${ }^{2 \star}$
}

\begin{abstract}
Quarks cannot be observed as free particles in nature because they are confined into baryons and mesons, as a result of the fact that the strong interaction between them increases with their separation. However, it is less known that this phenomenon also occurs in condensed matter and statistical physics as experimentally proved in several quasi-1D compounds ${ }^{1,2}$. Most of the theoretical and experimental studies so far concentrated on understanding the consequences of confinement for the equilibrium physics of both high-energy and condensed matter systems. Here, instead we show that confinement has dramatic consequences for the non-equilibrium dynamics following a quantum quench and that these effects could be exploited as a quantitative probe of confinement.
\end{abstract}

A global quantum quench is the non-equilibrium dynamics initiated by a sudden change of a parameter in the Hamiltonian of an isolated quantum system, a protocol that is routinely engineered in cold-atom experiments ${ }^{3-11}$. According to a by now standard picture $^{12}$, the initial state acts as a source of quasiparticle excitations. A quasiparticle of momentum $p$ moves with velocity $v_{p}$ and carries quantum correlations through the systems. If there is a maximum speed of propagation $v_{\max } \geq v_{p}$ (for example, as a consequence of the Lieb-Robinson bound $\left.{ }^{13}\right)$, all connected correlations at distance $\ell$ vanish for times $t<\ell / 2 v_{\max }$ (ref. 12) and the entanglement entropy of an interval of length $\ell$ grows linearly in the same time window ${ }^{14}$.

How can confinement change qualitatively the spreading of correlations? Exactly as in the standard scenario, the initial state emits pairs of quasiparticles starting off in opposite directions. However, these are eventually turned back by the confining potential, leading to an oscillatory behaviour as shown in Fig. 1. In analogy to strong interaction, the bound states formed by this process are called mesons. Mesons produced after a quench typically move much slower than the elementary quasiparticles due to their larger mass.

Confinement takes place in one of the paradigmatic models of statistical mechanics, the Ising chain in both transverse $\left(h_{z}\right)$ and longitudinal $\left(h_{x}\right)$ magnetic fields with Hamiltonian

$$
H=-J \sum_{j=-\infty}^{\infty}\left[\sigma_{j}^{x} \sigma_{j+1}^{x}+h_{z} \sigma_{j}^{z}+h_{x} \sigma_{j}^{x}\right]
$$

where the coupling $J$ sets the energy scale and $\sigma_{j}^{\alpha}$ are the Pauli matrices. This model has been engineered with cold atoms ${ }^{15}$ and quench protocols have also been implemented ${ }^{9}$.

For $h_{x}=0$, the Hamiltonian (1) is diagonalized by a mapping to free fermions (see Supplementary Information). For $h_{z}<1$ the system is in the ferromagnetic phase where the massive fermions are freely propagating domain walls separating domains of opposite magnetization. Switching on a non-zero $h_{x}$ induces a linear potential between pairs of domain walls confining them into mesons ${ }^{16}$. In this Letter, we report numerical results for several observables showing that the non-equilibrium quench dynamics is drastically altered by confinement.

\section{Expectation value of the order parameter}

We first consider the time evolution of the magnetization $\left\langle\sigma^{x}(t)\right\rangle$ that in the integrable case $\left(h_{x}=0\right)$ decays exponentially to zero ${ }^{17}$ (see Supplementary Information). For non-zero $h_{x}$, we report the numerical data for $\left\langle\sigma^{x}(t)\right\rangle$ in Fig. 2 for two representative quenches. It is evident that even a small longitudinal field alters substantially the dynamics, turning the exponential relaxation into an oscillatory behaviour with numerous frequencies ${ }^{18}$. The power spectrum presented in Fig. 2 shows that the dominant frequencies are compatible, to a surprising high degree of accuracy, with the masses of the mesons and their differences.

The two-point function shows the clearest signature of confinement manifested in a dramatically altered light-cone structure. In Fig. 3a we report the equal-time connected correlation function $\left\langle\sigma_{1}^{x} \sigma_{m+1}^{x}\right\rangle_{c}$ for the quench from the fully ferromagnetic state to $h_{z}=0.25$ and varying $h_{x}$ from 0 to 0.4 . If $h_{x}=0$, we recover the integrable dynamics with a clear light-cone spreading. For small $h_{x}=0.025$ the correlation follows qualitatively the integrable

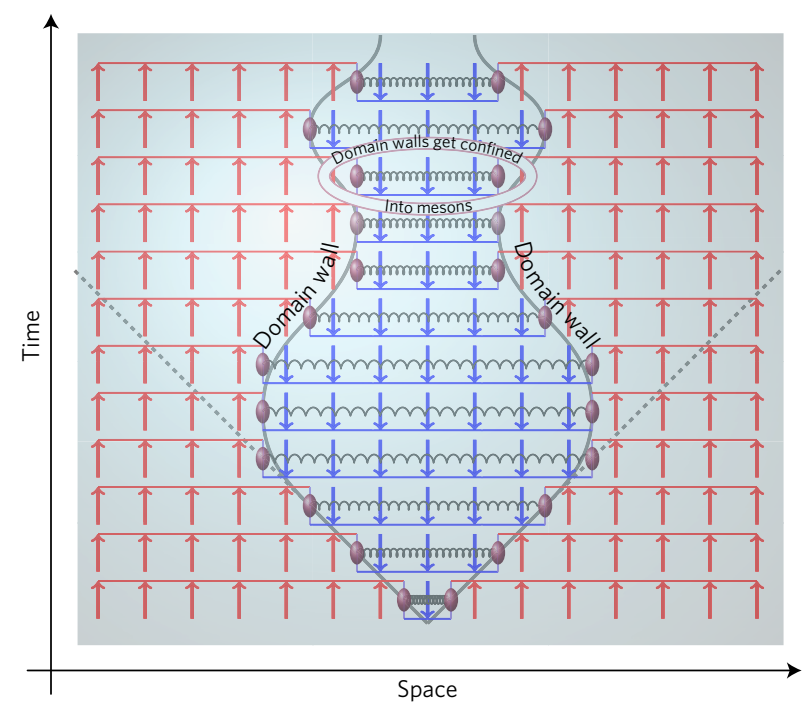

Figure 1 | Pictorial semiclassical picture of a meson state in the Ising model. Two counter-propagating domain walls bounce back and forth because of a confining interaction.

\footnotetext{
${ }^{1}$ MTA-BME "Momentum" Statistical Field Theory Research Group, 1111 Budapest, Budafoki út 8, Hungary. ${ }^{2}$ SISSA and INFN, via Bonomea 265, 34136 Trieste, Italy. ${ }^{3}$ Department of Theoretical Physics, Budapest University of Technology and Economics, 1111 Budapest, Budafoki út 8 , Hungary. *e-mail: calabrese@sissa.it
} 


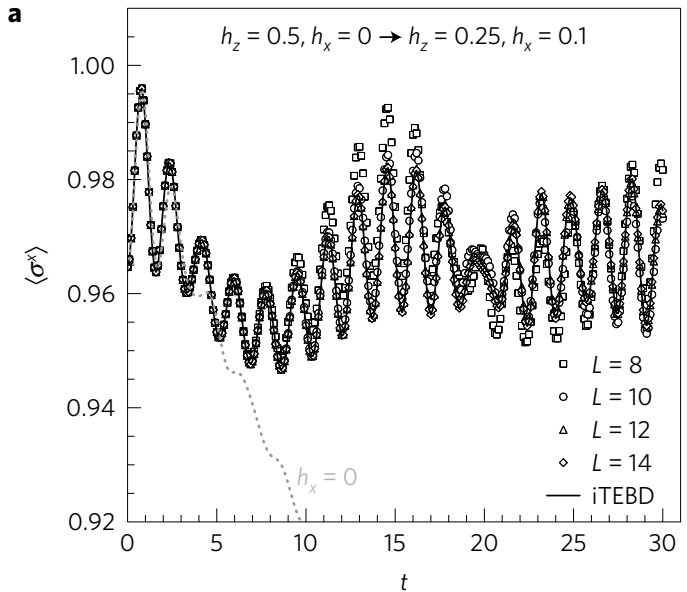

C

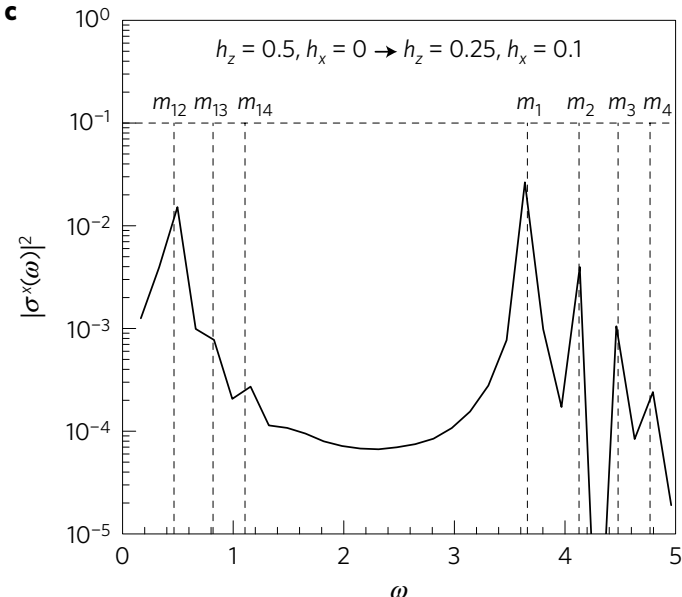

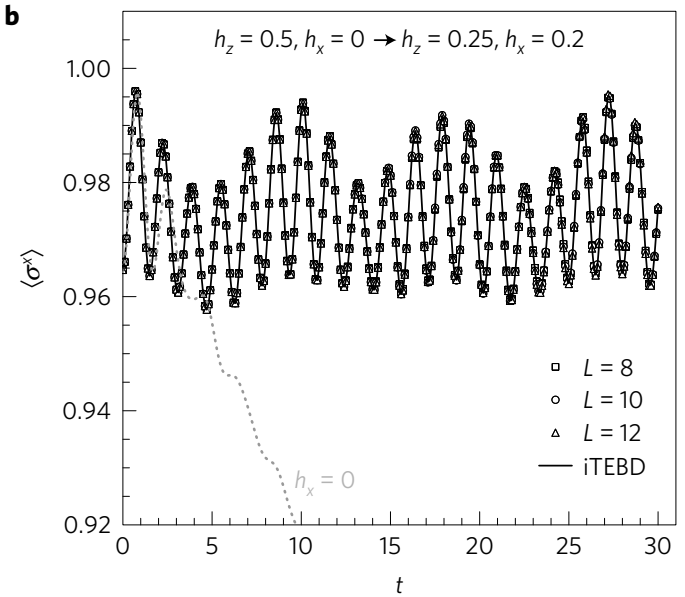

d

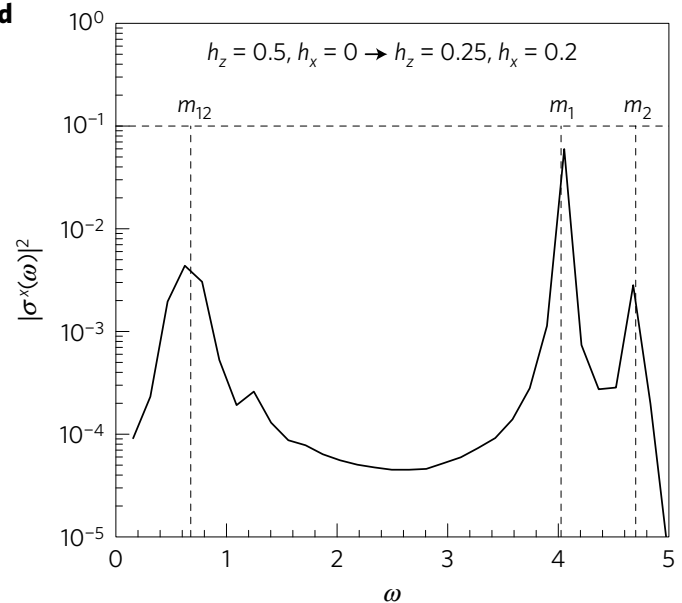

Figure 2 | Time evolution of the longitudinal magnetization. $\mathbf{a}, \mathbf{b},\left\langle\sigma^{x}(t)\right\rangle$ after quenching from $h_{z}=0.5, h_{x}=0$ to $h_{z}=0.25$ and $h_{x}=0.1,0.2$ (the result for $h_{x}=0$ is shown for comparison). Lines are iTEBD results; symbols are exact diagonalization results for $L=8, \ldots, 12$. Notice the weakness of finite-size effects: the iTEBD data for infinite chains are almost indistinguishable (up to a given time) from the exact diagonalization results. $\mathbf{c}, \mathbf{d}$, Power spectrum of the data in $\mathbf{a}$ and $\mathbf{b}$. The vertical lines show the meson masses $m_{i}$ and their differences $m_{i j}$.

behaviour for short times, but then it drastically slows down and bounces back. Further increasing $h_{x}$, the region with light-cone propagation shrinks to an almost invisible portion of the spacetime. This effect can be explained by considering that for a quench with $h_{x}=0$ the initial state can be written as ${ }^{17}$

$$
\left|\psi_{0}\right\rangle=\prod_{k>0}\left(1+i K(k) a_{k}^{\dagger} a_{-k}^{\dagger}\right)|0\rangle
$$

in terms of the post-quench domain wall excitation $a_{k}^{\dagger}$. This formula quantifies the fact that the initial state can be written in terms of pairs of particles with opposite momenta and $K(k)$ is the amplitude of production of these pairs (the exact dependence of $K(k)$ on $k$ is given in Supplementary Information, but this is inessential for the following). Adding a field $h_{x}$ confines the domain walls into mesons. When $K(k)$ is small, the state is dominated by the linear terms that contain only $(k,-k)$ domain walls pairs that get confined into mesons at rest. Quadratic terms (and higher order ones) lead to propagating mesons, but they are substantial only when $K(k)$ is large enough. These have velocities that are significantly different from those of the domain walls (see Supplementary Information).

For the quench in Fig. $3 \mathrm{a}, K(k) \ll 1$ for all momenta and practically only zero-momentum mesons are formed. Zooming in the 'white' region apparently without signal in Fig. 3a, traces of mesons with non-zero velocities should be visible. This is done in Fig. $3 \mathrm{~b}$ where the same connected correlation is displayed on a different intensity scale. The signal in Fig. $3 \mathrm{~b}$ is three orders of magnitude smaller than that in Fig. 3a and shows a feeble light cone characterized by a velocity different from that of the domain walls. The maximum meson velocity describes incredibly well the slope of the light cone for all values of $h_{x}$.

As further supporting evidence for the above scenario, in Fig. $3 \mathrm{c}$ we report connected correlations for a large quench (with $K(k) \gg 1$ ) from the paramagnetic phase to the ferromagnetic confining one. In this case, given that mesons with non-zero velocities are formed with high probability, the light cones are visible without zooming, and their slopes correspond to the maximal velocity of the mesons. A crucial consistency check of the validity of the overall scenario is that for quenches to the paramagnetic (non-confining) phase with $h_{x} \neq 0$ there is no radical change in the light cone as shown in Fig. $3 \mathrm{~d}$.

Similarly, the connected correlation function of the transverse component of the spin also reflects the change of the light cone due to the modified velocity of the mesons (see Supplementary Information). Furthermore, for quenches from and to several other values of the magnetic fields the overall picture is unchanged, so our conclusions are very general and not limited to the reported cases.

Entanglement entropy provides another smoking gun for the quasiparticle propagation and light-cone effects ${ }^{14}$. For zero $h_{x}$, we observe in Fig. 4 a linear growth in time of the half-chain entanglement entropy in agreement with the known exact results ${ }^{19}$. By turning on the interaction $h_{x}$, the growth of the entanglement entropy for quenches within the ferromagnetic phase is strongly slowed down and, after a transient, it appears to oscillate around a 

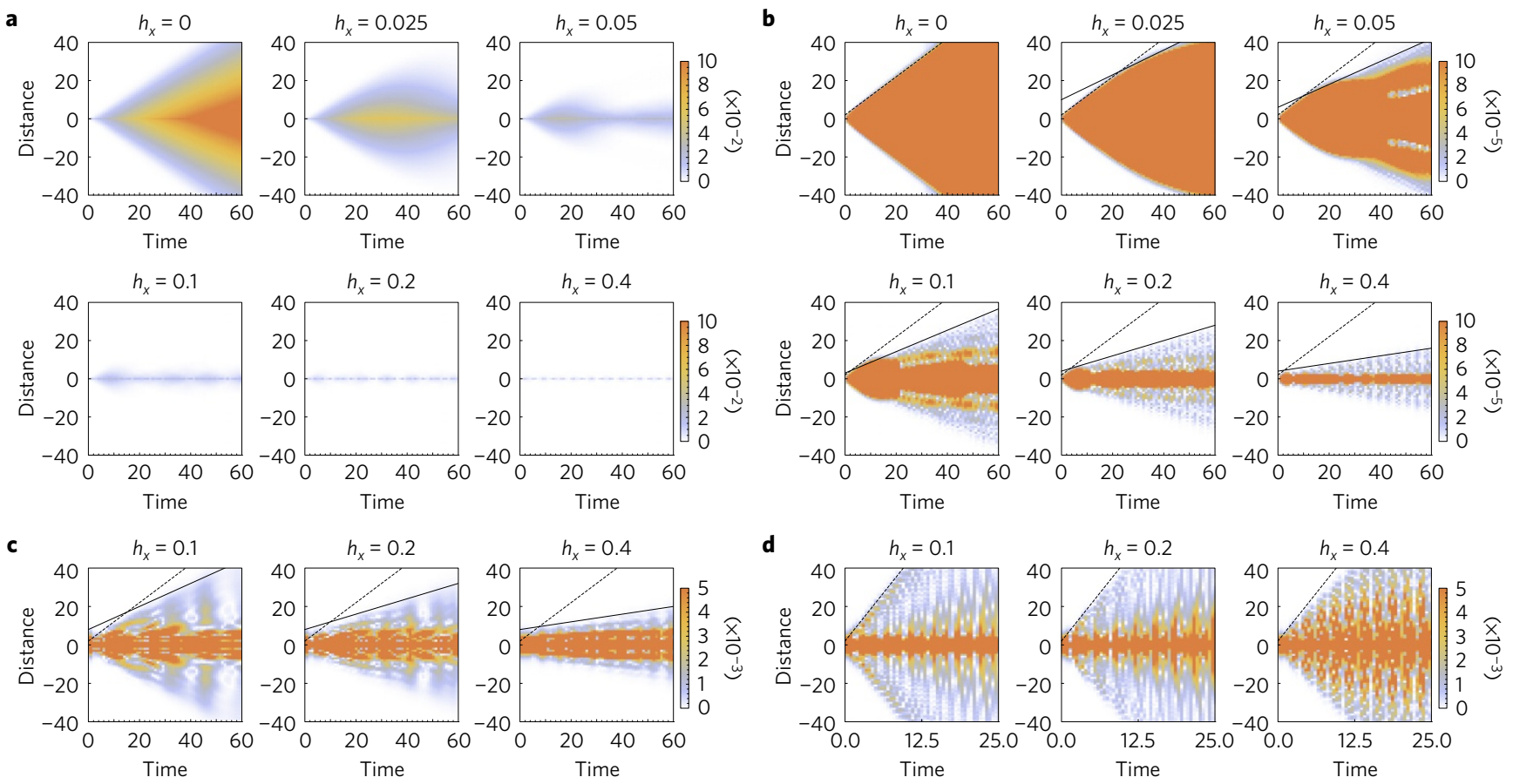

d
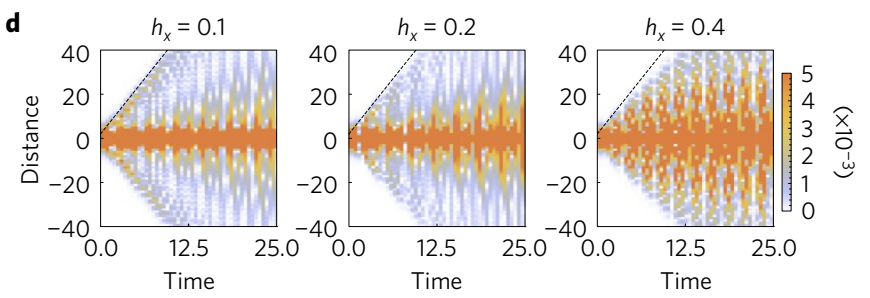

Figure 3 | Connected longitudinal spin-spin correlation function $\left\langle\sigma_{1}^{x} \sigma_{m+1}^{x}\right\rangle_{c}$. a, Quench from $h_{x}=h_{z}=0$ to the ferromagnetic phase with $h_{z}=0.25$ and

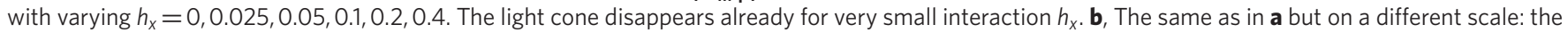

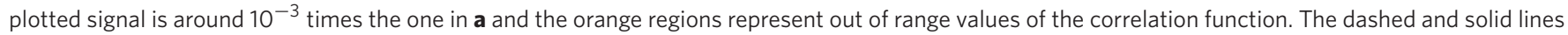
correspond to the maximal velocity of the domain walls and of the lightest meson, respectively. c, Absolute value of the correlation function for a quench from the paramagnetic phase $\left(h_{z}=2, h_{x}=0\right)$ to the ferromagnetic one $\left(h_{z}=0.25\right.$, varying $\left.h_{x}\right)$. d, Quench within the paramagnetic phase from $h_{z}=2$ and $h_{x}=0$ to $h_{z}=1.75$ and varying $h_{x}$. While in the confining phase the light cone experiences a drastic non-perturbative change, in the paramagnetic phase it is only perturbatively modified.
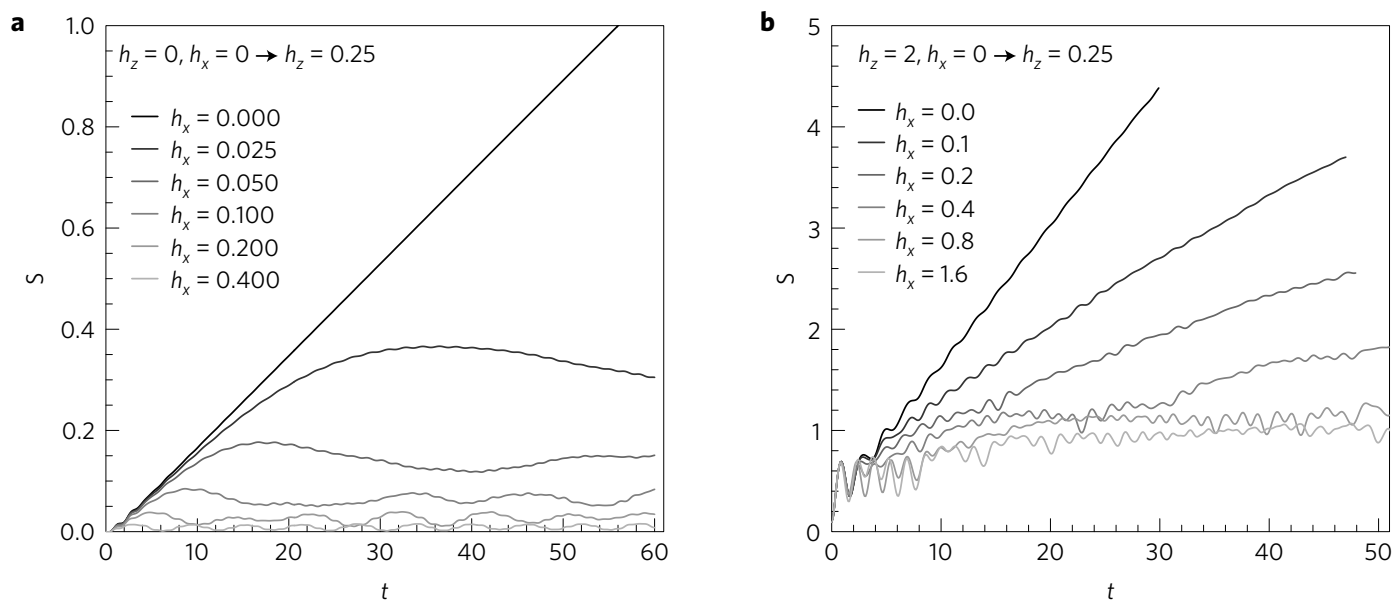

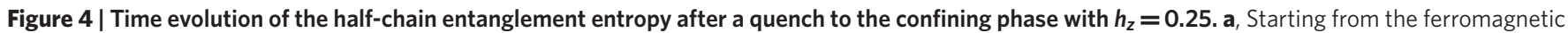

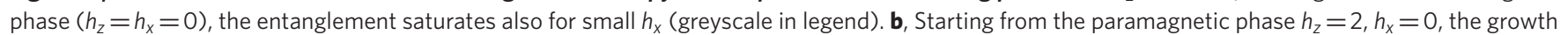
of the entanglement entropy is considerably reduced, and for very large $h_{x}$ it also saturates.

saturation value. The frequencies of these oscillations are in rough agreement with the meson masses and their differences as reported in Supplementary Information. This is a consequence of the fact that mesons are predominantly produced at rest and it is consistent with the strong suppression of the light cone of the two-point function. Actually, the small fraction of mesons with non-negligible velocities should produce a very slow increase of the entanglement that, however, is too small to be observed numerically. Conversely, for a quench across the critical point, the growth of the entanglement entropy is not negligible, but it is considerably reduced due to the production of many mesons with non-vanishing velocities. Overall, the data for the entanglement are compatible with the confinement scenario drawn for the correlations.

\section{Discussions}

We have given compelling arguments and numerical evidence proving that confinement strongly affects the non-equilibrium dynamics following a quantum quench. The main effect is a dramatic change of the light-cone structure of correlation functions and entanglement entropy. At the same time, the one-point function oscillates in time with frequencies equal to the meson masses. These effects should be easily measurable in cold-atom experiments; corrections 
due to the trapping harmonic potential are expected to be as small as the finite-size effects we observed. We also showed that the quench dynamics provides direct access to the meson masses via the power spectrum of the one-point functions. This 'quench spectroscopy' could turn out to be more powerful than standard equilibrium methods to measure the spectrum.

To conclude, we speculate on a few other consequences and applications of our work. It was noticed some time ago $^{20}$ that in some quenches within the Hamiltonian (1) the system does not approach asymptotically a thermal stationary state as expected from the nonintegrability of the model. A possible explanation is that due to confinement there are rare states in the spectrum that prevent the eigenstate thermalization hypothesis ${ }^{21}$ from being applied. Along the same line of thought, even if these confined systems eventually thermalize, the standard prethermalization scenario ${ }^{22,23}$ for weak integrability breaking cannot be applied, since a small perturbation changes not only the long-time asymptotic expectation values, but completely alters the dynamics even at short timescales.

Finally, confinement is expected to have similar effects also in higher dimensions and so it is natural to wonder what the consequences are for realistic non-equilibrium situations in quantum chromodynamics such as the quark-gluon plasma in hadron colliders. While even approximate field-theoretical calculations for strong interactions are beyond our reach, holographic methods that have been successfully applied to the study of standard lightcone phenomena ${ }^{24}$ and to quenches in confining theories ${ }^{25}$ could be insightful to understand this fascinating problem.

\section{Methods}

Methods, including statements of data availability and any associated accession codes and references, are available in the online version of this paper.

\section{Received 2 May 2016; accepted 21 September 2016;} published online 7 November 2016

\section{References}

1. Lake, B. et al. Confinement of fractional quantum number particles in a condensed-matter system. Nat. Phys. 6, 50-55 (2009).

2. Coldea, R. et al. Quantum criticality in an Ising chain: experimental evidence for emergent E8 symmetry. Science 327, 177-180 (2010).

3. Cheneau, M. et al. Light-cone-like spreading of correlations in a quantum many-body system. Nature 481, 484-487 (2012).

4. Kaufman, A. M. et al. Quantum thermalization through entanglement in an isolated many-body system. Science 353, 794-800 (2016).

5. Kinoshita, T., Wenger, T. \& Weiss, D. S. A quantum Newton's cradle. Nature 440, 900-903 (2006).

6. Hofferberth, S., Lesanovsky, I., Fischer, B., Schumm, T. \& Schmiedmayer, J. Non-equilibrium coherence dynamics in one-dimensional Bose gases. Nature 449, 324-327 (2007).

7. Trotzky, S. et al. Probing the relaxation towards equilibrium in an isolated strongly correlated 1D Bose gas. Nat. Phys. 8, 325-330 (2012).

8. Langen, T. et al. Experimental observation of a generalized Gibbs ensemble. Science 348, 207-211 (2015).
9. Meinert, F. et al. Many-body quantum quench in an atomic one-dimensional Ising chain. Phys. Rev. Lett. 111, 053003 (2013).

10. Fukuhara, T. et al. Microscopic observation of magnon bound states and their dynamics. Nature 502, 76-79 (2013).

11. Langen, T., Geiger, R., Kuhnert, M., Rauer, B. \& Schmiedmayer, J. Local emergence of thermal correlations in an isolated quantum many-body system. Nat. Phys. 9, 640-643 (2013).

12. Calabrese, P. \& Cardy, J. Time-dependence of correlation functions following a quantum quench. Phys. Rev. Lett. 96, 136801 (2006).

13. Lieb, E. H. \& Robinson, D. W. The finite group velocity of quantum spin systems. Commun. Math. Phys. 28, 251-257 (1972).

14. Calabrese, P. \& Cardy, J. Evolution of entanglement entropy in one dimensional systems. J. Stat. Mech. 0504, P04010 (2005).

15. Simon, J. et al. Quantum simulation of antiferromagnetic spin chains in an optical lattice. Nature 472, 307-312 (2011).

16. McCoy, B. M. \& Wu, T. T. Two-dimensional Ising field theory in a magnetic field: breakup of the cut in the two-point function. Phys. Rev. D 18, 1259-1267 (1978).

17. Calabrese, P., Essler, F. H. L. \& Fagotti, M. Quantum quench in the transverse field Ising chain. Phys. Rev. Lett. 106, 227203 (2011).

18. Delfino, G. Quantum quenches with integrable pre-quench dynamics. J. Phys. A 47, 402001 (2014).

19. Fagotti, M. \& Calabrese, P. Evolution of entanglement entropy following a quantum quench: analytic results for the XY chain in a transverse magnetic field. Phys. Rev. A 78, 010306 (2008).

20. Banuls, M. C., Cirac, J. I. \& Hastings, M. B. Strong and weak thermalization of infinite non-integrable quantum systems. Phys. Rev. Lett. 106, 050405 (2011).

21. D’Alessio, L., Kafri, Y., Polkovnikov, A. \& Rigol, M. From quantum chaos and eigenstate thermalization to statistical mechanics and thermodynamics. Adv. Phys. 65, 239-362 (2016).

22. Gring, M. et al. Relaxation and prethermalization in an isolated quantum system. Science 337, 1318-1322 (2012).

23. Bertini, B., Essler, F. H. L., Groha, S. \& Robinson, N. J. Prethermalization and thermalization in models with weak integrability breaking. Phys. Rev. Lett. 115, 180601 (2015).

24. Balasubramanian, V. et al. Staessens, thermalization of strongly coupled field theories. Phys. Rev. Lett. 106, 191601 (2011).

25. Buchel, A., Heller, M. P. \& Myers, R. C. Equilibration rates in a strongly coupled nonconformal quark-gluon plasma. Phys. Rev. Lett. 114, 251601 (2015).

\section{Acknowledgements}

We are grateful to R. M. Konik and J. Cardy for helpful discussions. This work was supported by the ERC under Starting Grant 279391 EDEQS (P.C. and M.C.). M.K. and G.T. thank SISSA for hospitality. M.K. was partially supported by a Janos Bolyai Research Scholarship of the HAS. This work was also partially supported by the CNR-HAS bilateral grant SNK-84/2013.

\section{Author contributions}

All authors contributed to the numerical simulations, the interpretation of the results, developing of the theory and to the writing of the manuscript.

\section{Additional information}

Supplementary information is available in the online version of the paper. Reprints and permissions information is available online at www.nature.com/reprints.

Correspondence and requests for materials should be addressed to P.C.

\section{Competing financial interests}

The authors declare no competing financial interests. 


\section{Methods}

The Hamiltonian (1) for $h_{x} \neq 0$ and $h_{z} \neq 0$ is not integrable and the spectrum can be described only by resorting to various approximations, such as, for example, field-theoretical ones ${ }^{26-28}$ that are valid in the vicinity of the critical point $h_{z}=1$ and $h_{x}=0$. Here we use a complementary approach: the low-density approximation of ref. 29, which describes the energy levels very accurately when the system is far away from the critical point. In the Supplementary Information we explain how this approach works and we report several pieces of evidence for its applicability for the values of magnetic fields of interest. This approximation allows us to calculate all the required properties of the mesons, namely their number, masses and velocities.

To simulate the time evolution after the quantum quench we use an iTEBD ${ }^{30}$ algorithm, details of which are reported in the Supplementary Information. Finally, the entanglement entropy is defined as the von Neumann entropy $S_{A}=-\operatorname{Tr} \rho_{A} \ln \rho_{A}$ of the reduced density matrix $\rho_{A}$ of a subsystem $A$.
Data availability. The data that support the plots within this paper and other findings of this study are available from the authors on request.

\section{References}

26. Mussardo, G. Integrability, non-integrability and confinement. J. Stat. Mech. 1101, P01002 (2011).

27. Delfino, G. Integrable field theory and critical phenomena: the Ising model in a magnetic field. J. Phys. A 37, R45 (2004).

28. Fonseca, P. \& Zamolodchikov, A. Ising spectroscopy I: mesons at $T<T_{c}$. Preprint at http://arXiv.org/abs/hep-th/0612304 (2006).

29. Rutkevich, S. B. Energy spectrum of bound-spinons in the quantum Ising spin-chain ferromagnet. J. Stat. Phys. 131, 917-939 (2008).

30. Vidal, G. Classical simulation of infinite-size quantum lattice systems in one spatial dimension. Phys. Rev. Lett. 98, 070201 (2007). 